Pacific Journal of Mathematics

UNICOHERENT COMPACTIFICATIONS

ward Clamp and Raymond Frank Dickman 


\title{
UNICOHERENT COMPACTIFICATIONS
}

\author{
M. H. Clapp and R. F. Dickman, JR.
}

In this paper we give necessary and sufficient conditions for the Freudenthal compactification of a rimcompact, locally connected and connected Hausdorff space to be unicoherent. We give several necessary and sufficient conditions for a locally connected generalized continuum to have a unicoherent compactification and show that if such a space $X$ has a unicoherent compactification, then $\gamma X$ is the smallest unicoherent compactification of $X$ in the usual ordering of compactifications.

A connected topological space $X$ is said to be unicoherent if, $H \cdot K$ is connected whenever $X=H+K$ where $H$ and $K$ are closed connected sets. A continuum is a compact connected metric space and a generalized continuum is a locally compact, connected, separable metric space. By a mapping we will always mean a continuous function. If $B$ is a subset of a space $X$, the closure of $B$ in $X$ will be denoted by $\mathrm{cl}_{X} B$ and the boundary of $B$ in $X$ will be denoted by $\operatorname{Fr}_{X} B$. An open set (respectively, a closed set) of a space $X$ will be called a $\gamma$-open (respectively, $\gamma$-closed) subset of $X$ provided it has a compact boundary in $X$. A space is rimcompact (or semicompact) provided every point has arbitrarily small neighborhoods with compact boundaries. All compactifications considered here are Hausdorff.

In [7] $\mathrm{K}$. Morita showed that for any rimcompact Hausdorff space $X$ there exists a topologically unique compactification $\gamma X$ of $X$ satisfying:

(a) For every point $x$ of $\gamma X$ and every open set $R$ of $\gamma X$ containing $x$ there exists an open set $V$ of $\gamma X$ containing $x$ such that $V \subset R$ and $\mathrm{Fr}_{\gamma X} V \subset X$.

(b) Any two disjoint $\gamma$-closed subsets of $X$ have disjoint closures in $\gamma X$.

Furthermore if $C$ is any compactification of $X$ satisfying (a), there exists a mapping $h$ of $\gamma X$ onto $C$ such that $h \mid X$ is the identity map. The compactification $\gamma X$ of $X$ is called the Freudenthal compactification of $X$ after H. Freudenthal who first defined it [4].

Definition. We say that a connected space $X$ is $\gamma$-unicoherent if whenever $X=H+K$, where $H$ and $K$ are $\gamma$-closed and connected sets, $H \cdot K$ is connected.

THeOREm 1. If $X$ is a locally connected, connected, rimcompact Hausdorff space, then $\gamma X$, the Freudenthal compactification of $X$, is 
unicoherent iff $X$ is $\gamma$-unicoherent.

Proof. Suppose that $X$ is $\gamma$-unicoherent and $\gamma X$ is not unicoherent. Then $\gamma X=H+K$ where $H$ and $K$ are closed and connected sets and $H \cdot K$ is not connected. Let $H \cdot K=A+B$ be a separation of $H \cdot K$ and let $U$ and $V$ be open subsets of $\gamma X$ containing $A$ and $B$ respectively such that $\mathrm{cl}_{\gamma X} U \cdot \mathrm{cl}_{\gamma X} V=\Phi$ and $\left(\mathrm{Fr}_{\gamma X} V+\mathrm{Fr}_{\gamma X} U\right) \subset X$. By Propositions (2.8) and (4.1) of [1], $\gamma X$ is locally connected so if $C$ denotes the component of $U+V+H$ that contains $H$ and $D$ denotes the component of $U+V+K$ that contains $K, C$ and $D$ are open connected subsets of $\gamma X$ such that $\left(F r_{\gamma X} C+\operatorname{Fr}_{\gamma X} D\right) \subset X$. By Lemma 5 of [6], $C \cdot X$ and $D \cdot X$ are connected so that $L=\operatorname{cl}_{X}(C \cdot X)$ and $M=$ $\operatorname{cl}_{X}(D \cdot X)$ are $\gamma$-closed and connected subsets of $X$. Furthermore $X=$ $L+M$ and $L \cdot M$ is not connected. This contradicts our hypothesis that $X$ is $\gamma$-unicoherent and thus $\gamma X$ must be unicoherent.

Now suppose that $\gamma X$ is unicoherent and $X$ is not $\gamma$-unicoherent. Then $X=H+K$ where $H$ and $K$ are $\gamma$-closed and connected subsets of $X$ and $H \cdot K$ is not connected. Let $H \cdot K=A+B$ be a separation of $H \cdot K$ and let $H^{\prime}, K^{\prime}, A^{\prime}$ and $B^{\prime}$ denote the closures of $H, K, A$ and $B$, respectively, in $\gamma X$. Since the boundary of $H \cdot K$ in $X$ is a subset of the union of the boundaries of $H$ and $K$ in $X, H \cdot K$ and hence $A$ and $B$ are $\gamma$-closed subsets of $X$. Then by property (b) of Morita's characterization of $\gamma X, A^{\prime}$ and $B^{\prime}$ are disjoint closed subsets of $\gamma X$. We now argue that $H^{\prime} \cdot K^{\prime}$ is a subset of $A^{\prime}+B^{\prime}$. Suppose to the contrary that there exists a point $x$ in $H^{\prime} \cdot K^{\prime}$ that does not belong to $A^{\prime}+B^{\prime}$. Let $U$ be any open subsets of $\gamma X$ containing $x$ such that $U$ does not intersect $A^{\prime}+B^{\prime}$ and such that $\operatorname{Fr}_{r X} \subset X$. Let $Q$ be the component of $U$ that contains $x$ and note that $\operatorname{Fr}_{\gamma X} Q$ is a subset of $X$ and $Q$ is an open subset of $\gamma X$. Then since $X$ is dense in $\gamma X$ and $x$ is a limit point of $H^{\prime}$ and $K^{\prime}, Q \cdot H$ and $Q \cdot K$ are nonempty sets. But by Lemma 5 of [6], $Q \cdot X$ is connected and since $Q$ misses $H \cdot K, Q \cdot X$ must lie entirely in $H$ or $K$. Of course this implies that either $Q \cdot H$ or $Q \cdot K$ is empty and this is a contradiction. Thus $H^{\prime} \cdot K^{\prime}=A^{\prime}+B^{\prime}$ and this contradicts the unicoherence of $\gamma X$. Therefore $X$ is $\gamma$ unicoherent.

We need the following notation and definitions. Let $S^{1}$ denote the unit circle in the complex plane, let $I_{1}=\left\{z=e^{i \theta}: 0 \leqq \theta \leqq \Pi\right\}$ and let $I_{2}=\left\{z=e^{i \theta}: \Pi \leqq \theta \leqq 2 \Pi\right\}$. For any space $W$ let $\mathscr{A}(W)$ denote the set of mappings of $W$ into $S^{1}$ and let $\mathscr{A}_{j}(W)$ be the set of all mappings of $W$ into $I_{j}, j=1,2$. For each $f \in \mathscr{A}_{j}(W), j=1,2$, let $B_{j}(f)$ denote the set of all points $t \in I_{j}$ such that $\operatorname{Fr} f^{-1}(t)$ contains a compact set $K$ that separates $W$ into two disjoint open sets $M$ and $N$ where $f$ maps $M$ into the arc from 1 to $t$ on $I_{j}$ and $f$ maps $N$ into the arc from $t$ to -1 on $I_{j}$. Finally let $E(W)=\left\{f \in \mathscr{A}(W): B_{1}\left(f \mid f^{-1}\left(I_{1}\right)\right)+\right.$ 
$B_{2}\left(f \mid f^{-1}\left(I_{2}\right)\right)$ is dense in $\left.S^{1}\right\}$.

Theorem 2. Suppose that $X$ is a locally connected, rimcompact Hausdorff space. A necessary and sufficient condition that $\gamma X$ be unicoherent is that every element of $E(X)$ be nullhomotopic.

Proof of the necessity. Suppose that $\gamma X$ is unicoherent and let $f$ be an element of $E(X)$. For $j=1,2$, there exists a point $t_{j} \in I_{j}$ such that $\operatorname{Fr}_{X} f^{-1}\left(t_{j}\right)$ contains a compact set $K_{j}$ that separates $f^{-1}\left(I_{j}\right)$ into two disjoint open sets $M_{j}$ and $N_{j}$ where $f$ maps $M_{j}$ into the arc from 1 to $t_{j}$ on $I_{j}$ and $f$ maps $N_{j}$ into the arc from $t_{j}$ to -1 on $I_{j}$. Then if we let $M$ denote $K_{1}+K_{2}+M_{1}+M_{2}$ and let $N$ denote $K_{1}+K_{2}$ $N_{1}+N_{2}, X=M+N$ and the boundaries (relative to $X$ ) of $M$ and $N$ are subsets of $K=K_{1}+K_{2}$. We assert that the boundaries of $M_{0}=\operatorname{cl}_{\gamma X} M$ and $N_{0}=\operatorname{cl}_{\gamma X} N$ relative to $\gamma X$ are also subsets of $K$. In order to see this suppose that $x$ is an element of the boundary of $M_{0}$ and $x \notin$ $K$. Then since $\gamma X$ is locally connected, there exists an open connected set $R$ of $\gamma X$ containing $x$ such that $R \cdot K=\Phi$ and $\mathrm{Fr}_{\gamma X} R \subset X$. Then $R \cdot M \neq \Phi$ and $R \cdot(X \backslash M) \neq \Phi$ since $X$ is dense in $\gamma X$. Furthermore $R \cdot X$ is connected by Lemma 5 of [6] and so $R \cdot X$ is a connected subset of $X$ that meets $M$ and $X \backslash M$. This implies that $R$ meets $K$ and this contradicts our selection of $x$. Hence the boundaries of $M_{0}$ and $N_{0}$ in $\gamma X$ are subsets of $K$. Also by Theorem 3 of [7], $M_{0}$ and $N_{0}$ are topologically equivalent to $\gamma M$ and $\gamma N$ respectively. Then by Lemma 1 of [3], $f \mid M$ has a continuous extension $f_{M}$ to $M_{0}$ and $f \mid N$ has a continuous extension $f_{N}$ to $N_{0}$. Then since $N_{0} \cdot M_{0} \subset$ $K$, the function $h$ of $\gamma X$ into $S^{1}$ defined by $h \mid M_{0}=f_{M}$ and $h \mid N_{0}=$ $f_{N}$ is continuous. By Lemma (7.4) of [9, p. 228], $h$ is exponentially representable on $\gamma X$, i.e. there exists a real valued function $\theta$ on $\gamma X$ such that $h(x)=e^{i \theta(x)}$ for all $x \in X$. It is evident that this implies that $f=h \mid X$ is exponentially representable an $X$ and by Theorem (6.2) of $[9$, p. 226], $f$ is nullhomotopic.

Proof of the sufficiency. Suppose that every element of $E(X)$ is nullhomotopic and suppose that $\gamma X$ is not unicoherent. Then by the proof of Theorem 1 there exists closed and connected sets $H$ and $K$ of $\gamma X$ such that $H \cdot K$ is not connected, Fr $H$ and Fr $K$ are subsets of $X$ and $L=H \cdot X$ and $M=K \cdot X$ are connected. Let $H \cdot K=A+$ $B$ be a separation of $H \cdot K$. We note that $L$ and $M$ are $\gamma$-closed subsets of and thus by Theorem 3 of [7], $\gamma L$ is homeomorphic to $H$ and $\gamma M$ is homeomorphic to $K$. It then follows from Lemma 2 of [3] that there exists a mapping $f$ of $H$ into $I_{1}$ such that $f(A)=1, f(B)=-1$ and $B_{1}(f \mid H \cdot X)$ is dense in $I_{1}$. Similarly there exists a mapping $g$ of $K$ into $I_{2}$ such that $g(A)=1, g(B)=-1$ and $B_{2}(g \mid K \cdot X)$ is dense 
in $I_{2}$. Then if we define $h: \gamma X \rightarrow S^{1}$ by $h \mid H=f$ and $h \mid K=g$ we have that $h$ is continuous and $k=h \mid X$ is an element of $E(X)$. Then by our hypothesis and Proposition 6.2 of [9, p. 226], $k$ is exponentially representable, i.e. there exists a real-valued mapping $\theta$ on $X$ such that for each $x \in X, k(x)=e^{i \theta(x)}$. But then $\theta(A) \subset\{0, \pm 2 \Pi, \pm 4 \Pi, \cdots\}$ and $\theta(B) \subset\{ \pm \Pi, \pm 3 \Pi, \cdots\}$ and so if $a \in \theta(A)$ and $b \in \theta(B)$, the interval $[a, b]$ lies in $\theta(A) \cdot \theta(B)$ since $L$ and $M$ are connected. This is a contradiction since then $k(L) \cdot k(M)$ would then contain a semicircle whereas it consists of the points -1 and 1 . Hence $\gamma X$ is unicoherent.

DEFINITION. A connected space $X$ is said to be weakly unicoherent if whenever $X=H+K$ where $H$ and $K$ are closed and connected sets and $K$ is compact, $H \cdot K$ is connected.

Theorem 3. Let $X$ be a locally connected generalized continuum. $A$ necessary and sufficient condition for $\gamma X$ to be unicoherent is that $X$ be weakly-unicoherent.

Proof of the necessity. Suppose that $\gamma X$ is unicoherent. Since $X$ is locally compact, $X$ is open in $\gamma X$ and $X^{*}=\gamma X \backslash X$ is closed. Then by Theorem (2.3) of [2], $X=\gamma X \backslash X^{*}$ is weakly-unicoherent.

Proof of the sufficiency. Suppose that $\gamma X$ is not unicoherent. Then as in the proof of Theorem $1, \gamma X$ has a representation $\gamma X=$ $P+Q$ where $P$ and $Q$ are open connected subsets of $\gamma X$, the boundaries of $P$ and $Q$ in $\gamma X$ are subsets of $X, \operatorname{cl}_{\gamma x} P \cdot \operatorname{cl}_{\gamma x} Q=A+B$ where $A$ and $B$ are disjoint nonempty closed sets and $P$ has a nonempty intersection with both the boundary of $A$ and the boundary of $B$. By Lemma 5 of [6], $P^{\prime}=P \cdot X$ is a connected open subset of $X$ and thus is arcwise connected. Furthermore since the boundaries of $A$ and $B$ are subsets of $X$ there exists an arc $\alpha \beta$ in $P^{\prime}$ such that $\alpha \beta \cdot A=$ $\alpha$ and $\alpha \beta \cdot B=\beta$. Let $R$ be the component of $P^{\prime} \backslash(A+B)$ that contains $\alpha \beta \backslash(\alpha+\beta)$ and let $W$ be an open subset of $\gamma X$ containing $A$ such that $B \cdot \operatorname{cl} W=\phi$ and the boundary of $W$ is a subset of $X$. Then $H=$ $R \cdot \mathrm{Fr}_{r x} W$ is a nonempty compact subset of $R$ and there exists a continuum $K_{0}$ of $X$ such that $H \subset K_{0} \subset R$. Let $K$ be the union of $K_{0}$ together with all the components of $R \backslash K_{0}$ with boundary entirely in $K_{0}$, i.e. having no boundary points in $X \cdot(A+B)$. Then $K$ separates $R$ since $W \cdot R$ contains a subarc $\alpha b \backslash \alpha$ from some point $b \in \alpha \beta$ and $X \backslash \mathrm{cl}_{x} W$ contains a subarc $\alpha \beta$ of $\alpha \beta$. But $X \backslash K$ is connected since $X \backslash K$ is the union of the closure of $Q$ in $X$ plus all of the components of $X \backslash(A \cdot B)$ except $R$ plus all of the components of $R-K_{0}$ having a boundary point in $X \cdot(A+B)$. This contradicts Whyburn's characterization of weak-unicoherence in [8, p. 185]. 
Corollary 3.1. Let $X$ be a locally connected generalized continuum. Then $X$ is weakly-unicoherent iff $X$ is $\gamma$-unicoherent.

This corollary follows immediately from Theorems 1 and 3 .

REMARK. The authors have been unable to discover a direct proof of Corollary (3.1). In general the two types of unicoherency are not equivalent and in the absence of local compactness, Theorem 3 is not valid.

ExAMPLE. Let $Y=\{z$ complex $|1 / 2 \leqq| z \mid \leqq 1\}$,

$$
\begin{aligned}
S & =\{z|| z \mid=1\}, A \text { a countable dense subset of } S, \\
L_{z} & =Y \cdot\{\text { ray from origin thru } z\} \\
C_{r} & =\{z|| z \mid=r\}, r \in[1 / 2,1] ; \\
Z & =\left\{C_{r} \cdot L_{a} \mid r \text { is rational, } a \in A\right\} .
\end{aligned}
$$

The set $Z$ is countable and dense in $Y$. Let $X=Y-Z$. The set $X$ is evidently $T_{2}$, connected and locally connected (in fact, path connected and locally path connected), rim compact but not locally compact. Moreover:

1. $X$ is weakly-unichoherent. To see this, note that any continuum $K \subset X$ has empty interior in $X$. If therefore $X=H+K, H$ closed and connected and $K$ compact and connected, then necessarily the open set $X-H$ is a subset of $K$, and thus empty. It follows that $H \cdot K=K$, which is connected.

2. $X$ is not $\gamma$-unicoherent. For let $p, q \in S-A$ be two distinct points. Then $L_{p}$ and $L_{q}$ are compact and disjoint subsets of $X$. Assume $0 \leqq A R G p<A R G q$. Then

$$
\begin{aligned}
& H=\left\{z \in X \mid A R G p \leqq A R G z \leqq A R G_{q}\right\} \text { and } \\
& K=\left\{z \in X \mid A R G q \leqq A R G_{z} \leqq A R G p+2 \pi\right\}
\end{aligned}
$$

are closed, connected subsets of $X$ such that $X=H+K, H \cdot K=L_{p}+$ $L_{q}$ is compact but not connected.

3. $\gamma X$ is not unicoherent. To show this it is sufficient to show that $\gamma X$ is just the set $Y$. To this end we use the characterization of $\gamma X$ obtained by Morita [6]. We show that

(a) For any point $x \in \gamma X$ and open set $R$ of $\gamma X$ containing $x$, there is an open set $V$ of $r X$ containing $x$ such that $V \subset R$ and $\operatorname{Fr}_{r X} V \subset X$.

(b) Any two disjoint $\gamma$-closed subsets of $X$ have disjoint closures in $\gamma X$. 
That (a) holds is evident from the definition of $X$. To see that (b) holds, let $A$ and $B$ be disjoint $\gamma$-closed subsets of $X$ and suppose that $p \in \operatorname{cl}_{\gamma_{X}} A \cdot \operatorname{cl}_{\gamma_{X}} B$. First of all we note that $p$ cannot belong: to $X$ for then it would lie in $A \cdot B$ which is empty. In particular $p$ does not lie in the compact set $\left(\operatorname{Fr}_{X} A+\mathrm{Fr}_{X} B\right)$. By our construction of $X$ there exists an open subset $V$ of $Y$ containing $p$ such that $V \cdot\left(\operatorname{Fr}_{X} A+\operatorname{Fr}_{X} B\right)=\Phi$ and $V \cdot X$ is connected. Since $p$ belongs to the closure of $A$ in $Y, V \cdot X \cdot A$ is not empty and since $V \cdot X$ misses $\operatorname{Fr}_{X} A$, $V \cdot X$ must lie entirely in $A$. But this is a contradiction since $V \cdot X$ must meet $B$. Therefore $A$ and $B$ have disjoint closures in $Y$.

Definition. A mapping $f: X \in Y$ is monotone provided for every $y \in Y, f^{-1}(y)$ is compact and connected.

THEOREM 4. If $X$ is a locally connected generalized continuum and $Y$ is any unicoherent compactification of $X$, then there exists a monotone mapping $g$ of $Y$ onto $\gamma X$ such that $g \mid X$ is the identity.

Proof. Let $Z$ denote the quotient space of $Y$ obtained from the decomposition whose only nondegenerate elements are the components of $Y \backslash X$ and let $p$ denote the natural map of $Y$ onto $Z$. Then since $X$ is open in $Y, Z$ is a Hausdorff compactification of $X$. Furthermore since point inverses of $p$ are connected, it follows from Proposition (2.2.1) of [9], that $Z$ is unicoherent. Also $Z \backslash X$ is totally disconnected and by the maximality of $\gamma X$ there exists a mapping $h$ of $\gamma X$ onto $Z$ such that $h \mid X$ is the identity and $h(\gamma X \backslash X)=Z \backslash X$. We assert that $h$ is a homeomorphism. In order to prove this we need only show that $h$ is one-to-one on $\gamma X \backslash X$. To this end let $x, y \in \gamma X, x \neq y$ and suppose that $h(x)=h(y)$. There exists a connected and open set $R$ of $\gamma X$ containing $x$ such that $y \notin \mathrm{cl}_{\gamma} R=K$ and $\operatorname{Fr}_{\gamma} R \subset X$. Then $Z=$ $h(K)+h(\gamma X \backslash R)$ and $h(K) \cdot h(\gamma X \backslash R)=h(x)+h(\operatorname{Fr} R)$ is not connected. This contradicts the unicoherence of $Z$ and hence $h$ must be a homeomorphism. Then $g=h^{-1} \circ p$ is the desired monotone mapping.

Corollary 4.1. Suppose that $X$ is a locally connected generalized continuum. Then $X$ has a unicoherent compactification if and only if $\gamma X$ is unicoherent.

Proof. This result follows immediately from Theorem 4 and the fact that monotone images of compact unicoherent continua are unicoherent.

THEOREM 5. Suppose that $X$ is a locally connected generalized continuum. Then the following are equivalent 
(i) $X$ is weakly-unicoherent

(ii) $\gamma X$ is unicoherent

(iii) $X$ is $\gamma$-unicoherent

(iv) $X$ has a unicoherent compactification

(v) every mapping of $X$ into $S^{1}$ with compact boundaries of point inverses is null-homotopic.

Proof. The equivalence of (i)-(iv) has been established in Theorems (1) - (4). As an immediate consequence of Theorem (3.3) of [2], we have that (v) implies (i) and (ii) implies (v) follows from Theorem 1 of this paper.

Definition. A connected space $X$ is said to have the complementation property provided whenever $K$ is a compact set in $X, X / K$ has at most one component with a non-compact closure. See [2] for some characterizations of this property.

THEOREM 6. Let $X$ be a locally connected generalized continuum and let $Y$ be any unicoherent, locally connected continuum. There exists a unicoherent compactification $Z$ of $X$ with $Z \backslash X$ homeomorphic to $Y$ if and only if $X$ is weakly-unicoherent and has the complementation property.

Proof of the necessity. Suppose that $Z$ is a unicoherent compactification of $X$ and $Z \backslash X$ is homeomorphic to $Y$. Then by Theorem (4.2) of [2], $X$ is weakly-unicoherent and has the complementation property.

Proof of the sufficiency. Suppose that $X$ is weaklyunicoherent and has the complementation property. Then by Theorem (2.2) of [5] there exists a compactification $Z$ of $X$ with $Z \backslash X$ homeomorphic to $Y$ and by Theorem (4.2) of [2], $Z$ is unicoherent. This completes the proof.

REMARK. It appears to be difficult to establish results concerning the unicoherence of a compactification of an arbitrary completely regular space. We can show that the Freudenthal compactification of a rim-compact, locally connected $\gamma$-unicoherent space is the smallest unicoherent compactification of $X$ with $\gamma X \backslash X$ zero-dimensional.

\section{REFERENCES}

1. J. DeGroot and R. H. McDowell, Locally connected spaces and their compactifications, Illinois J. Math., 11 (1967), 353-364. 
2. R. F. Dickman, Jr., Unicoherence and related properties, Duke Math. J., 34 (1967), $343-352$.

3. - Some characterizations of the Freudenthal compactification of a semicompact space, Proc. Amer. Math. Soc., 19 (1968), 631-633.

4. H. Freudenthal, Neuaufbau der Endentheorie, Ann. of Math., (2), 43 (1942), 261-279.

5. K. D. Magill, Jr., A note on compactifications, Math Zeitschr., 94 (1966), 322-325.

6. K. Morita, On images of an open interval under closed continuous mappings, Proc. Japan Acad., 32 (1959), 15-19.

7. — On bicompactifications of semibicompact spaces, Sci. Rep. Tokyo Bunrika Daigaku Sect. A, 4 (1952), 222-229.

8. G. T. Whyburn, Open mapings on 2-dimensional manifolds, J. Math. Mech., 10 (1961), 181-198.

9. —, Analytic Topology, Amer. Math. Soc. Colloq. Pub., Vol. 28 (1942).

Received April 27, 1971.

California State College at Fullerton

AND

Virginia Polytechnic Institute and

StATE UNIVERSity 


\section{PACIFIC JOURNAL OF MATHEMATICS}

\section{EDITORS}

\section{H. SAMElson}

Stanford University

Stanford, California 94305

C. R. Новву

University of Washington

Seattle, Washington 98105
J. DugunduI

Department of Mathematics University of Southern California

Los Angeles, California 90007

RICHARD ARENS

University of California

Los Angeles, California 90024

\section{ASSOCIATE EDITORS}
E. F. BECKENBACH
B. H. NeUmanN
F. WOLF
K. YosHIDA

\section{SUPPORTING INSTITUTIONS}

UNIVERSITY OF BRITISH COLUMBIA

UNIVERSITY OF SOUTHERN CALIFORNIA

CALIFORNIA INSTITUTE OF TECHNOLOGY

STANFORD UNIVERSITY

UNIVERSITY OF CALIFORNIA

UNIVERSITY OF TOKYO

MONTANA STATE UNIVERSITY

UNIVERSITY OF UTAH

UNIVERSITY OF NEVADA

WASHINGTON STATE UNIVERSITY

NEW MEXICO STATE UNIVERSITY

OREGON STATE UNIVERSITY

UNIVERSITY OF OREGON

OSAKA UNIVERSITY

UNIVERSITY OF WASHINGTON

* *

AMERICAN MATHEMATICAL SOCIETY

NAVAL WEAPONS CENTER 


\section{Pacific Journal of Mathematics}

\section{Vol. 43, No. $1 \quad$ March, 1972}

Alexander (Smbat) Abian, The use of mitotic ordinals in cardinal

arithmetic ....................................... 1

Helen Elizabeth. Adams, Filtrations and valuations on rings ......... 7

Benno Artmann, Geometric aspects of primary lattices .............. 15

Marilyn Breen, Determining a polytope by Radon partitions ........... 27

David S. Browder, Derived algebras in $L_{1}$ of a compact group .......... 39

Aiden A. Bruen, Unimbeddable nets of small deficiency .............. 51

Michael Howard Clapp and Raymond Frank Dickman, Unicoherent

compactifications ............................... 55

Heron S. Collins and Robert A. Fontenot, Approximate identities and the strict topology ................................... 63

R. J. Gazik, Convergence in spaces of subsets................. 81

Joan Geramita, Automorphisms on cylindrical semigroups ........... 93

Kenneth R. Goodearl, Distributing tensor product over direct product ..... 107

Julien O. Hennefeld, The non-conjugacy of certain algebras of

operators ................................... 111

C. Ward Henson, The nonstandard hulls of a uniform space ........... 115

M. Jeanette Huebener, Complementation in the lattice of regular

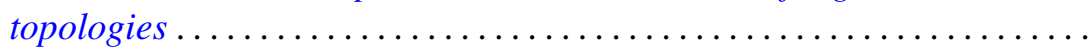

Dennis Lee Johnson, The diophantine problem $Y^{2}-X^{3}=A$ in a

polynomial ring .................................... 151

Albert Joseph Karam, Strong Lie ideals . . . . . . . . . . . . . . . . . . . . 157

Soon-Kyu Kim, On low dimensional minimal sets ............... 171

Thomas Latimer Kriete, III and Marvin Rosenblum, A Phragmén-Lindelöf

theorem with applications to $M(u, v)$ functions ..... . .

William A. Lampe, Notes on related structures of a universal algebra . . . . 189

Theodore Windle Palmer, The reducing ideal is a radical .

207

Kulumani M. Rangaswamy and N. Vanaja, Quasi projectives in abelian and module categories ................................ 221

Ghulam M. Shah, On the univalence of some analytic functions ......... 239

Joseph Earl Valentine and Stanley G. Wayment, Criteria for Banach

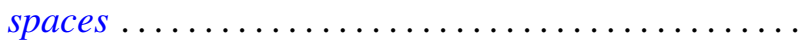

Jerry Eugene Vaughan, Linearly stratifiable spaces ............... 253

Zbigniew Zielezny, On spaces of distributions strongly regular with respect to partial differential operators ..................... 